\title{
Taking in consideration the bystander effects of articular senescence
}

\author{
Jean-Marc Brondello, Yves-Marie Pers \\ IRMB, University of Montpellier, Inserm U1183, CHU Montpellier, Montpellier, France \\ Correspondence to: Yves-Marie Pers, MD, PhD. IRMB, University of Montpellier, Inserm U1183, CHU Montpellier, 371, avenue du doyen Gaston \\ Giraud; 34295 Montpellier, France. Email: ym-pers@chu-montpellier.fr. \\ Provenance: This is an invited article commissioned by the Section Editor Dr. Yu Zhang (Department of Sport Medicine and Adult Reconstructive \\ Surgery, Drum Tower Hospital, School of Medicine, Nanjing University, Nanjing, China). \\ Comment on: Cao X, Luo P, Huang J, et al. Intraarticular senescent chondrocytes impair the cartilage regeneration capacity of mesenchymal stem \\ cells. Stem Cell Res Ther 2019;10:86.
}

Submitted Dec 07, 2019. Accepted for publication Dec 18, 2019.

doi: 10.21037/atm.2019.12.128

View this article at: http://dx.doi.org/10.21037/atm.2019.12.128

Osteoarthritis (OA) is a whole-joint disease and one of the leading causes of chronic pain and physical disability through the world $(1,2)$. Its prevalence is increasing drastically with the aging population (3). Causes of OA are multifactorial: genetic predisposition, alteration of joint component, aging, and mechanical factors (obesity, joint misalignment and joint trauma). $\mathrm{OA}$ is characterized by progressive deterioration and loss of joint cartilage combined with anatomical and molecular alterations of other joint structures. In particular, OA leads to a loss of chondrocyte function that is overcome during the repair process of cartilage damage. There is an abnormal differentiation of normal chondrocytes into hypertrophic chondrocytes. The latter contribute to the breakdown mechanisms through the production of pro-inflammatory mediators and accelerated aging.

Cellular senescence is a state in which cell proliferation is blunt through the expression of cell cycle inhibitors concomitantly to the establishment of one tissue remodeling secretome called Senescent Associated Secretory Phenotype (SASP) proficient to propagate senescence. Extrinsic cues for instance, DNA damaging agents or chronic inflammation but also intrinsic signals such as telomere shortening can induce such senescent phenotypes (4). Cellular senescence has thus many beneficial effects as tumour suppressive mechanisms as well as during embryonic development and wound healing. However, the persistence and the accumulation of such cells in various tissues during lifespan lead to chronic tissue degeneration and onset of many age-related diseases (5). Recent studies demonstrated that articular chondrocytes isolated from OA patients have senescent phenotypes by harboring shorten telomeres, by accumulating cycle-dependent kinases inhibitors driving senescence such as $\mathrm{p} 16^{\text {Ink4a }}, \mathrm{p} 21^{\text {Cdkn1A }}$ and $\mathrm{p} 57^{\mathrm{KIP} 2}$, and finally by producing deleterious catabolic and inflammatory mediators (6). Remarkably, the use of senolytic drug namely UBX0101 that specifically eliminates senescent cells in experimentally-induced OA murine models, delay cartilage degradation. For the first time, it was hence demonstrated that cellular senescence in joint tissues play a pivotal causal role in OA pathogenesis (7).

Current therapy of OA, without exception, are palliative. Clearly, there is an obvious medical need that would be best met by an effective, safe, well tolerated, local treatment with disease-modifying properties. As alternative, regenerative stem cell therapies based on Mesenchymal Stromal Cells (MSC) infusion in OA patient joints have been proposed (8). Indeed, MSC possess immunomodulatory and anti-fibrotic properties, can protect targeted cells from oxidative stress, senescence and even apoptotic onset. Meanwhile MSC, through paracrine properties, are believed to stimulate proliferation and differentiation of tissue resident stem cells (8). In OA patients, the safety of MSC local injection has been proven in many trials, but evidence of efficacy compared to currently proposed palliative treatments remains to be demonstrated (9). Thus, the low efficient 
regenerative potentials of injected MSC observed so far can possibly be related to the joint pro-senescent environment induced by $\mathrm{OA}$ disease. In line with this hypothesis, in the recent study, Cao and colleagues reveal the deleterious paracrine dialogues taking place between senescent chondrocytes and bone marrow derived MSC using rat model (10). In trans-well setting, irradiationinduced senescent chondrocytes modify the behaviour of co-cultivated MSCs by showing a dual-opposite effect depending on the timing. After 7 days, senescent chondrocytes prevent MSCs proliferation but increase the expression of chondrogenic markers such Sox 9, Col II. In contrast, at 21 days, senescent chondrocytes stimulate both senescence and apoptosis on MSC while decreasing their chondrogenic potentials. Surprisingly, MSCs induce, in turn, apoptosis on senescent chondrocytes confirming their paracrine seno-suppressive potentials. To confirm the causal effects of articular senescence on MSC altered functions, the authors pre-treated senescent chondrocytes with navitoclax, a BCL2/BCLXL inhibitory drug having senolytic properties on other senescent cell types (11). Although, in this study, no real demonstration has been done on the clearance efficacy of senescent chondrocytes by such concentration of navitoclax, Cao et al. data suggest that treated senescent chondrocytes restore MSC stemness and differentiation potentials compared to untreated senescent chondrocytes. Finally, through experimental-induced OA on rat models, they could confirm the in vivo benefit of such senolytic agent as a synergistic treatment, prior MSCs delivery, for the repair of focal cartilage lesions.

The study by Cao et al. also has many limitations. First, they used primary rat chondrocytes but at late passages (p3) which may induce a risk of chondrocyte de-differentiation. Second, they used irradiation to trigger senescence on chondrocytes, far from the normal pathophysiological process. Third, the biological effects of co-cultivated MSCs with senescent chondrocytes was often compared to MSCs alone and not to healthy chondrocytes. It would have been more appropriate, in the context of in vivo cell therapy using MSCs to compare them in the presence of healthy chondrocytes. Actually, the results seem quite similar on proliferation, apoptosis, or expression of MSC differentiation markers. However, a different in vitro biological effect with chondrogenesis at 21 days was observed since the expression of Col II and Aggrecan was not modified when MSC was co-cultivated in the presence or without senescent chondrocytes. Finally, if we consider that MSCs injected into the joint survive only a few hours in vivo (12), the dual-opposite effect according to the coculture time of senescent chondrocytes with MSCs reported by the authors does not seem critical to optimize the therapeutic effect of MSC in OA disease.

Altogether, this study supports the involvement of senescence in the pathophysiology of OA. The therapeutic use of senolytic drugs is currently under development in OA with promising results reported at the recent US Congress of Rheumatology (13). A further perspective would be to combine the two therapeutic options (senolytic and cellular therapy by MSC) in the treatment of OA but, nowadays, the biological relevance of this approach remains to be determined.

\section{Acknowledgments}

None.

\section{Footnote}

Conflicts of Interest: The authors have no conflicts of interest to declare.

Ethical Statement: The authors are accountable for all aspects of the work in ensuring that questions related to the accuracy or integrity of any part of the work are appropriately investigated and resolved.

\section{References}

1. Cross M, Smith E, Hoy D, et al. The global burden of hip and knee osteoarthritis: estimates from the global burden of disease 2010 study. Ann Rheum Dis 2014;73:1323-30.

2. Vos T, Flaxman AD, Naghavi M, et al. Years lived with disability (YLDs) for 1160 sequelae of 289 diseases and injuries 1990-2010: a systematic analysis for the Global Burden of Disease Study 2010. Lancet 2012;380:2163-96.

3. Martel-Pelletier J, Barr AJ, Cicuttini FM, et al. Osteoarthritis. Nat Rev Dis Prim 2016;2:16072.

4. He S, Sharpless NE. Senescence in Health and Disease. Cell 2017;169:1000-11.

5. Tachikart Y, Malaise O, Mumme M, et al. Senosuppressive molecules as new therapeutic perspectives in rheumatic diseases. Biochem Pharmacol 2019;165:126-33.

6. McCulloch K, Litherland GJ, Rai TS. Cellular senescence in osteoarthritis pathology. Aging Cell 2017;16:210-8.

7. Jeon $\mathrm{OH}$, Kim C, Laberge RM, et al. Local clearance of senescent cells attenuates the development of post- 
traumatic osteoarthritis and creates a pro-regenerative environment. Nat Med 2017;23:775-81.

8. Pers YM, Ruiz M, Noël D, et al. Mesenchymal stem cells for the management of inflammation in osteoarthritis: state of the art and perspectives. Osteoarthritis Cartilage 2015;23:2027-35.

9. Maumus M, Pers YM, Ruiz M, et al. Mesenchymal stem cells and regenerative medicine: future perspectives in osteoarthritis. Med Sci (Paris) 2018;34:1092-9.

10. Cao X, Luo P, Huang J, et al. Intraarticular senescent chondrocytes impair the cartilage regeneration capacity of mesenchymal stem cells. Stem Cell Res Ther 2019;10:86.

11. Zhu Y, Tchkonia T, Fuhrmann-Stroissnigg H, et al.

Cite this article as: Brondello JM, Pers YM. Taking in consideration the bystander effects of articular senescence. Ann Transl Med 2019;7(Suppl 8):S386. doi: 10.21037/ atm.2019.12.128
Identification of a novel senolytic agent, navitoclax, targeting the Bcl-2 family of anti-apoptotic factors. Aging Cell 2016;15:428-35.

12. Toupet K, Maumus M, Peyrafitte JA, et al. Long-term detection of human adipose-derived mesenchymal stem cells after intraarticular injection in SCID mice. Arthritis Rheum 2013;65:1786-94.

13. Hsu B, Visich J, Genovese M, et al. Safety, Tolerability, Pharmacokinetics, and Clinical Outcomes Following Single-Dose IA Administration of UBX0101, a Senolytic MDM2/p53 Interaction Inhibitor, in Patients with Knee OA. In: Abstract number L05. ACR/ARP Annual Meeting; 2019 Nov 8-13; Atlanta, USA. 